\title{
Efeito de 12 Semanas de Treinamento \\ Com Pesos Sobre a Força Muscular, Composição Corporal e Triglicérides \\ em Homens Sedentários
}

\section{2-Week Resistance Training Effect on Muscular Strength, Body Composition and Triglycerides in Sedentary Men}

Marcos Doederlein Polito 1,2 Edilson Serpeloni Cyrino, Aline Mendes Gerage ${ }^{1,2}$ Matheus Amarante do Nascimento ${ }^{2}$ Renata Selvatici Borges Januário 2,3

1. Grupo de Estudo e Pesquisa em Respostas Cardiovasculares e Exercício. Centro de Educação Física e Esporte. Universidade Estadual de Londrina.

2. Grupo de Estudo e Pesquisa em Metabolismo, Nutrição e Exercício. Centro de Educação Física e Esporte. Universidade Estadual de Londrina. 3. Departamento de Educação

Física. Universidade Norte do Paraná.

\section{Endereço para correspondência:} Grupo de Estudo e Pesquisa em Respostas Cardiovasculares e Exercício.

Centro de Educação Física e Esporte. Universidade Estadual de Londrina. Rod. Celso Garcia Cid, km 380 -

Campus Universitário. 86051-990 - Londrina, PR - Brasil. E-mail: marcospolito@uel.br

\section{RESUMO}

O objetivo do presente estudo foi verificar o efeito de 12 semanas de treinamento com pesos (TP) sobre a força muscular, composição corporal e triglicérides em homens sedentários. Para tanto, 14 homens saudáveis e sedentários foram separados aleatoriamente, de forma balanceada, em grupo experimental $(G E=29 \pm 1$ anos; 180,0 \pm 3,0cm; 80,5 \pm 1,8kg) e grupo controle (GC $=27 \pm 1$ anos; 170,0 $\pm 2,0 \mathrm{~cm} ; 76,2 \pm$ 0,9kg). O GE foi submetido a um protocolo de TP durante 12 semanas, com frequência de três vezes por semana. O programa de TP foi composto por 10 exercícios executados em duas séries de 10-20 repetições. Medidas de massa corporal, estatura, espessura de dobras cutâneas e triglicérides foram realizadas antes e após o período de intervenção. Adicionalmente, o teste de uma repetição máxima (1RM) foi aplicado nos exercícios supino em banco horizontal e mesa extensora, nos período pré, após seis e 12 semanas de acompanhamento. Foram encontradas diferenças significativas $(P<0,05)$ após as 12 semanas de TP apenas no GE para o somatório das dobras cutâneas, ao passo que a massa corporal e os triglicérides não sofreram alterações nesse período. Com relação aos valores de força muscular, identificou-se aumento significativo $(P<0,05)$ no GE em ambos os exercícios entre o primeiro e o segundo teste de 1 RM e entre o segundo e o terceiro. Conclui-se, portanto, que 12 semanas de TP foram suficientes para aumentar a força muscular e reduzir o somatório de dobras cutâneas sem, contudo, alterar os valores de triglicérides e massa corporal.

Palavras-chave: treinamento de força, triglicéride plasmático, percentual de gordura.

\section{ABSTRACT}

The purpose of this study was to investigate the effect of 12 weeks of resistance training on muscle strength, body composition and triglycerides in sedentary men. Fourteen healthy men were randomly divided into experimental ( $E G=29 \pm 1 \mathrm{yrs} ; 180.0 \pm 3.0 \mathrm{~cm} ; 80.5 \pm 1.8 \mathrm{~kg}$ ) and control $(C G=27 \pm 1 \mathrm{yrs}$; $170.0 \pm 2.0 \mathrm{~cm} ; 76.2 \pm 0.9 \mathrm{~kg}$ ) groups. The EG subjects participated in a resistance training protocol during 12 weeks, three times per week. The program of resistance training included 10 exercises performed in two sets of 10-20 repetitions. Body mass, height, skinfold thickness and triglycerides were measured before and after the 12 weeks of intervention. One maximal repetition test (1RM) was done to measure muscle strength. Significant differences $(P<0.05)$ were observed on the sum of skinfolds only in $E G$ after the resistance training, while body mass and triglycerides did not change in neither groups Muscle strength increased $(P<0.05)$ in both exercises performed between the first and the second and between the second and the third 1-RM tests. Therefore, 12 weeks of resistance training were enough to improve muscle strength and sum of skinfolds, but without promoting any changes in triglycerides and body mass.

Keywords: strength training, plasma triglycerides, body fat percentage. 


\section{INTRODUÇÃO}

Nos últimos anos, tem sido observado aumento acentuado no número de adeptos ao treinamento com pesos (TP), bem como no número de publicações investigando os efeitos desse tipo de treinamento sobre os diferentes componentes da aptidão física associada à saúde ou ao desempenho.

Nesse sentido, um dos principais benefícios decorrentes da prática de TP mostrados pela literatura está relacionado com o aumento da força, da resistência muscular e da massa corporal magra ${ }^{(1,2)}$, o que faz com que programas envolvendo esse tipo de treinamento sejam considerados como uma ferramenta bastante interessante a ser adotada por indivíduos de diferentes faixas etárias.

No entanto, diferenças metodológicas observadas em alguns estudos dificultam a determinação da magnitude desses benefícios. Por exemplo, no que diz respeito ao efeito desse modelo de exercício sobre a composição corporal, ainda existem controvérsias, uma vez que alguns pesquisadores advogam que o treinamento predominantemente aeróbio seria o mais adequado, sobretudo quando se objetiva a redução no percentual de gordura corporal ${ }^{(3,4)}$. O TP, por sua vez, caracterizado como atividade de alta intensidade, está intimamente relacionado com ganhos na massa muscular e, por isso, parece acarretar uma redução na gordura corporal, em virtude do aumento no gasto energético(5).

Além disso, mais recentemente, o TP tem se mostrado benéfico no controle de alguns fatores de risco para o desenvolvimento de doenças coronarianas $^{(6)}$. Aparentemente, influências positivas no perfil lipídico e lipoproteico são observadas em decorrência desse tipo de treinamento. Entretanto, isso ainda não está bem estabelecido na literatura, uma vez que muitos programas envolvendo o TP não têm demonstrado alterações nessa variável ${ }^{(4,7,8)}$.

Dessa forma, considerando potenciais lacunas, o objetivo do presente estudo foi verificar o efeito de 12 semanas de TP sobre a força muscular, composição corporal e triglicérides em homens sedentários.

\section{MÉTODOS}

\section{Amostra}

A amostra foi composta por 14 homens voluntários, aparentemente saudáveis e sedentários pelo menos seis meses antecedentes ao início do experimento. Os indivíduos foram distribuídos igualmente em grupo experimental (GE $=29 \pm 1$ anos; 180,0 $\pm 3,0 \mathrm{~cm} ; 80,5 \pm 3,8 \mathrm{~kg}$ ) e grupo controle (GC $=27 \pm 1$ anos; 170,0 $\pm 2,0 \mathrm{~cm} ; 79,7 \pm 2,4 \mathrm{~kg})$. Durante a aplicação do programa de TP, os grupos foram orientados a não realizar qualquer outro exercício físico, na tentativa de o impacto desse programa ser analisado de forma individual.

Como critérios de exclusão foram considerados tabagismo, uso de substâncias ergogênicas, doenças crônicas preexistentes e resposta positiva ao questionário Par-Q. Após receber informação sobre os procedimentos aos quais seriam submetidos, os sujeitos assinaram um termo de consentimento livre e esclarecido. Os procedimentos adotados neste estudo atenderam às normas da Resolução 196/96 do Conselho Nacional de Saúde para pesquisas em seres humanos, após aprovação do comitê de ética em pesquisa da Universidade Estadual de Londrina (Parecer 265/06).

\section{Medidas antropométricas e composição corporal}

Para a determinação da massa corporal e estatura foi utilizada uma balança mecânica acoplada a um estadiômetro (Welmy $\left.{ }^{\oplus}\right)$. As medidas foram realizadas de acordo com os procedimentos de Gordon et al..$^{(9)}$. A composição corporal foi determinada por meio da técnica de espessura das dobras cutâneas utilizando um compasso Lange, sendo utilizados os pontos anatômicos peitoral, abdominal e coxa, de acordo com o protocolo de Jackson e Pollock ${ }^{(10)}$.

\section{Coleta de sangue}

Os triglicérides plasmáticos foram analisados pelo método enzimático colorimétrico, após coleta de sangue de 10-12h em jejum. A fim de evitar interferências no experimento, os sujeitos foram instruídos a manter o mesmo padrão alimentar.

\section{Teste de uma repetição máxima (1RM)}

A força muscular foi determinada por meio do teste de 1RM. Os exercícios realizados foram o supino em banco horizontal e extensão de pernas, sendo a ordem de execução aleatória. Para cada exercício, os sujeitos tinham até cinco tentativas para a determinação de 1RM, com intervalo de três a cinco minutos entre as tentativas e os exercícios.

Anteriormente ao início do estudo, foi empregado um protocolo de familiarização ao teste de 1RM, na tentativa de reduzir os efeitos de aprendizagem aos gestos motores. Todos os sujeitos foram testados, em situação semelhante à do protocolo adotado, em três sessões distintas intervaladas por períodos de 48 horas.

\section{Protocolo de treinamento com pesos}

O GE realizou um programa de TP, três vezes por semana, durante 12 semanas, sendo que a frequência média dos sujeitos foi de 34 sessões de treinamento.

O programa foi constituído pelos exercícios realizados na seguinte ordem: supino em banco horizontal, extensão de pernas, supino inclinado, puxada no pulley, flexão de pernas, remada, tríceps no pulley, agachamento na máquina, desenvolvimento e rosca direta de bíceps. Esses indivíduos realizaram, inicialmente, duas séries de 15-20 repetições e intensidade determinada subjetivamente próxima à fadiga. A partir da terceira e quarta semana, foram realizadas 10-15 repetições com a mesma intensidade. O ajuste das cargas ocorria quando o sujeito reportava condições de realizar confortavelmente as repetições determinadas. Em todos os casos, o intervalo de recuperação entre as séries e os exercícios foi de 50-180s.

\section{Delineamento do estudo}

O estudo teve a duração total de 14 semanas. A primeira semana foi destinada à familiarização ao teste de 1RM, além da realização de anamnese, medidas antropométricas, avaliação da composição corporal e coletas de sangue. A partir da segunda semana, o GE foi submetido a um programa de TP com duração de 12 semanas.

As medidas da primeira semana foram realizadas em dois momentos distintos (M1 = início e M3 = 12 semanas após M1). O teste de 1RM, por sua vez, foi aplicado em três momentos (M1 = início, $M 2$ = seis semanas após $\mathrm{M} 1$ e M3 = 12 semanas após M1).

\section{Tratamento estatístico}

Os resultados são apresentados em média \pm erro padrão. As variáveis foram submetidas ao teste de Shapiro-Wilk para verificar a distribuição dos dados e ao teste de Levene, para investigar a homogeneidade das variâncias. Análise de variância (ANOVA) para medidas repetidas foi utilizada para verificar as possíveis modificações nos valores do teste de 1RM nos diferentes momentos (M1, M2, M3), seguida do teste post-hoc de Tukey. Utilizou-se o teste $t$ de Student para amostras dependentes com o objetivo de comparar os resultados das medidas antropométricas, composição corporal e triglicérides nos momentos M1 e M3. O nível de significância adotado foi de 5\% em todas as análises. Os cálculos foram realizados no programa Statistica versão 5.5 (Statsoft, Tulsa, OK, EUA). 


\section{RESULTADOS}

A tabela 1 apresenta as características antropométricas de ambos os grupos. Não foram observadas diferenças significativas para a massa corporal em nenhum dos grupos. No entanto, após 12 semanas de intervenção, o GE apresentou redução significativa no $\sum D C$, enquanto que para o GC houve aumento, apesar de não ser significativo.

Tabela 1. Comportamento da massa corporal e do somatório da espessura das dobras cutâneas peitoral, abdominal e coxa antes e após 12 semanas de acompanhamento, nos grupos experimental $(n=7)$ e controle $(n=7)$ (valores em média \pm erro padrão)

\begin{tabular}{|c|c|c|}
\hline Variáveis & Grupo experimental & Grupo controle \\
\hline \multicolumn{3}{|c|}{ Massa corporal (kg) } \\
\hline Pré & $80,5 \pm 3,8$ & $79,7 \pm 2,4$ \\
\hline Pós & $81,4 \pm 4,0$ & $82,1 \pm 2,9^{*}$ \\
\hline \multicolumn{3}{|l|}{$\Sigma \mathrm{DC}(\mathrm{mm})$} \\
\hline Pré & $89,8 \pm 11,3$ & $87,4 \pm 9,1$ \\
\hline Pós & $80,7 \pm 11,7^{*}$ & $90,0 \pm 8,7^{*}$ \\
\hline
\end{tabular}

Non

Nota: Os resultados estăo expressos em valores médios $( \pm \mathrm{DP})$.

Foi observado aumento significativo na força muscular (tabela 2) do M1 para o M2, M2 para M3 e M1 para M3 nos dois exercícios testados apenas no GE. O GC não apresentou modificações significativas na força muscular em nenhum dos momentos do estudo.

Tabela 2. Valores do teste de 1RM nos momentos M1 (início), M2 (seis semanas após M1) e M3 (12 semanas após M1) (média \pm erro padrão)

\begin{tabular}{|c|c|c|c|}
\hline Exercícios & M1 & M2 & M3 \\
\hline & \multicolumn{3}{|c|}{ Grupo experimental $(n=7)$} \\
\hline Supino em banco horizontal (kg) & $100,3 \pm 2,2^{*+}$ & $106,3 \pm 2,9^{\#}$ & $110,6 \pm 3,2$ \\
\hline \multirow[t]{2}{*}{ Extensão de pernas (kg) } & $78,3 \pm 3,7^{*+}$ & $94,0 \pm 5,5^{\#}$ & $102,9 \pm 5,2$ \\
\hline & \multicolumn{3}{|c|}{ Grupo controle $(n=7)$} \\
\hline Supino em banco horizontal (kg) & $98,2 \pm 3,2$ & $101,1 \pm 1,9$ & $103,5 \pm 2,1$ \\
\hline Extensão de pernas (kg) & $80,1 \pm 1,4$ & $83,3 \pm 2,2$ & $82,7 \pm 3,3$ \\
\hline
\end{tabular}

Em relação aos triglicérides, não foram identificadas diferenças significativas entre a medida inicial e a realizada no final do treinamento, tanto para o GE $\left(128,9 \pm 28,9\right.$ vs. $\left.103,1 \pm 20,4 \mathrm{mg} \mathrm{dL}^{-1}\right)$ quanto para o GC $\left(116,1 \pm 19,2\right.$ vs. $\left.135,8 \pm 27,2 \mathrm{mg}^{-\mathrm{dL}^{-1}}\right)$.

\section{DISCUSSÃO}

O presente estudo procurou investigar o efeito de 12 semanas de TP sobre a força muscular, composição corporal e triglicérides em homens sedentários. Os resultados apontaram aumento significativo de $6 \%$ na força muscular no exercício supino em banco horizontal e de $20 \%$ no exercício de extensão de pernas nas primeiras seis semanas para o GE. Ao final das 12 semanas, o aumento foi ainda maior (10\% para supino em banco horizontal e 31\% para extensão de pernas). Tais resultados corroboram outros experimentos disponíveis na literatura ${ }^{(1,2,11,12)}$.

Além disso, foi observada redução significativa no somatório de dobras cutâneas após as 12 semanas de intervenção no GE, ao passo que no GC houve aumento. Esses achados também estão de acordo com outros estudos publicados até o momento(5), embora esse ainda seja um ponto conflitante na literatura. Em relação às taxas de triglicérides, não foram encontradas diferenças significativas após o programa de TP. Esses resultados vão ao encontro de outras pesquisas que não apontam modificações significativas nessa variável em decorrência da prática de $\mathrm{TP}^{(4,8,13,14)}$.

No entanto, quando se trata de modificações no perfil lipídico como resultado de envolvimento em programas de TP, as informações presentes na literatura ainda não são consistentes. Algumas pesquisas demonstram alterações positivas nos lipídios sanguíneos ${ }^{(15,16)}$, enquanto outras não apresentam efeito significativo(17-19).

LeMura et al. ${ }^{(4)}$ investigaram o efeito de 16 semanas de diferentes modelos de exercício físico (aeróbio, TP e aeróbio mais TP) sobre as modificações no perfil lipídico, aptidão cardiovascular e composição corporal. Verificaram que o exercício predominantemente aeróbio meIhora o perfil lipídico, aumenta a aptidão cardiovascular e a composição corporal de mulheres jovens e saudáveis. O TP, por sua vez, apenas induziu aumentos na força muscular, tanto de membros inferiores quanto de membros superiores.

Da mesma forma, Banz et al. ${ }^{(13)}$, ao comparar o impacto do treinamento aeróbio e do TP sobre alguns fatores de risco para o desenvolvimento de doenças coronarianas em homens obesos, não observaram alterações nos valores de triglicérides no grupo que participou do programa de TP.

Fahlman et al.(20), ao examinar as modificações no perfil das lipoproteínas plasmáticas em 45 indivíduos saudáveis, aleatoriamente divididos em grupo treinamento aeróbio, TP e grupo controle, identificaram reduções, contudo, não significativas nos triglicérides tanto dos indivíduos que realizaram TP quanto nos que fizeram treinamento aeróbio, porém, o grupo controle apresentou aumento dos valores. Os autores apontam que as modificações nos níveis de triglicérides podem estar relacionadas com a intensidade do treinamento. Nesse sentido, Elliot et al.(21) investigaram o efeito de oito semanas de TP de baixa intensidade e oito semanas de destreinamento, no perfil lipídico de mulheres saudáveis pós-menopausa. Tanto a intervenção quanto o destreinamento não evidenciaram alterações significativas no perfil lipídico, sugerindo que o TP de baixa intensidade não foi suficiente para proporcionar alterações no perfil lipídico de mulheres com essas características.

Em contrapartida, um estudo publicado recentemente, investigando o efeito do TP sobre o perfil lipídico e a distribuição da gordura corporal em idosos, identificou reduções significativas nas taxas de triglicérides após 12 semanas de intervenção. No entanto, as sessões de treinamento não foram devidamente supervisionadas, o que poderia trazer importantes implicações aos resultados, principalmente por não ser possível determinar a intensidade em que os sujeitos treinaram, o que pode influenciar as modificações na variável em questão(22).

Alguns estudos sugerem que a redução das taxas de triglicérides após um período de prática de TP pode estar relacionada com o aumento de massa muscular induzido por esse tipo de treinamento(23). Todavia, no presente estudo, devido à técnica utilizada para a avaliação da composição corporal, não existem informações referentes à massa muscular dos sujeitos.

Kelley e Kelley ${ }^{(24)}$, em uma revisão com meta-análise, envolvendo 29 ensaios clínicos aleatórios, sugeriram que o TP reduz o colesterol total, a razão CT/HDL-C, não HDL-C (CT - HDL) e as taxas de triglicérides em adultos. Entretanto, essa revisão contradiz os resultados de outras duas revisões que indicaram que o TP proporciona pequena ou nenhuma redução no perfil lipídico ou lipoproteico de adultos ${ }^{(6,25)}$. Tais contradições presentes na literatura acerca da relação TP e taxas de 
triglicérides podem ser explicadas pelas diferenças na intensidade dos programas adotados, na duração das sessões, na frequência semanal de treino, na duração total do programa, no estado de treinamento ou nos níveis lipídicos pré-treinamento dos sujeitos envolvidos nos experimentos ${ }^{(26)}$.

A literatura indica que o nível inicial dos lipídios séricos influencia os efeitos que a nutrição e a atividade física podem exercer sobre os componentes lipídicos, sendo que indivíduos com níveis lipídicos mais elevados, usualmente, possuem maiores decréscimos por $\mathrm{mg} / \mathrm{dL}^{(27)}$. Isso pode justificar as modificações não significativas nos triglicérides encontradas no presente estudo, uma vez que os participantes estavam, antes do início do treinamento, com a média dos triglicérides enquadrada em um padrão considerado normal (menor que $150 \mathrm{mg} / \mathrm{dL}$ ).

Vale destacar que alguns estudos podem ter fracassado na tentativa de encontrar modificações das variáveis sanguíneas após um período de intervenção, especialmente pela falta de controle de algumas variáveis como ingestão dietética, o que pode ser considerado, talvez, a maior limitação da presente investigação.
Assim, sugere-se que estudos futuros atentem para esse fato na tentativa de que sejam mais bem compreendidos os reais efeitos desse tipo de treinamento, sobretudo, sobre o perfil lipídico, levando-se em consideração principalmente que a diminuição de triglicérides reduz o risco de doença cardiovascular em 7\% nas mulheres e 3\% nos homens ${ }^{(28)}$.

\section{CONCLUSÃO}

Com base nos resultados, conclui-se que 12 semanas de TP foram suficientes para aumentar a força muscular e reduzir o somatório de dobras cutâneas sem, contudo, alterar os valores de triglicérides e massa corporal.

\section{AGRADECIMENTOS}

A Andresa Dalsotto e Régis Muniz pelo auxílio na coleta de dados.

Todos os autores declararam não haver qualquer potencial conflito de interesses referente a este artigo.

\section{REFERÊNCIAS BIBLIOGRÁFICAS}

1. Humburg H, Baars H, Schröder J, Reer R, Braumann KM. 1-set vs. 3-set resistance training: a crossover study. J Strength Cond Res 2007;21:578-82.

2. Dias RMR, Cyrino ES, Salvador EP, Nakamura FY, Pina FLC, Oliveira AR. Impacto de oito semanas de treinamento com pesos sobre a força muscular de homens e mulheres. Rev Bras Med Esporte 2005;11:224-8.

3. Clinical guidelines on the identification, evaluation, and treatment of overweight and obesity in adults: the evidence report. National Institutes of Health. Obes Res 1998;6(Suppl 2):51S-2095.

4. LeMura LM, Duvillard SP, Adreacci J, Klebez JM, Chelland AS, Russo J. Lipid and lipoprotein profiles, cardiovascular fitness, body composition and diet during and after resistance, aerobic and combination training in young women. Eur J Appl Physiol 2000;82:451-8.

5. Dolezal BA, Potteiger JA. Concurrent resistance and endurance training influence basal metabolic rate in nondieting individuals. J Appl Physiol 1998;85:695-700.

6. Braith RW, Stewart KJ. Resistance exercise training: its role in the prevention of cardiovascular disease. Circulation 2006;113:2642-50.

7. Vincent KR, Braith RW, Bottiglieri T, Vincent HK, Lowenthal DT. Homocysteine and lipoprotein levels following resistance training in older adults. Prev Cardiol 2003;6:197-203.

8. Volek JS, Duncan ND, Mazzetti SA, Putukian M, Gomez AL, Kraemer WJ. No effect of heavy resistance training and creatine supplementation on blood lipids. Int J Sport Nutr Exerc Metab 2000;10:144-56.

9. Gordon CC, Chumlea WC, Roche AF. Stature, recumbent length, and weight. In: Lohman TG, Roche AF, Martorell R, editors. Anthropometric standardizing reference manual. Champaign: Human Kinetics Books, 1988;3-8.

10. Jackson AS, Pollock ML. Generalized equations for predicting body density of men. Br J Nutr 1978;40:497-504

11. Candow DG, Burke DG. Effect of short-term equal-volume resistance training with different workout frequency on muscle mass and strength in untrained men and women. J Strength Cond Res 2007;21:204-7.

12. Nóbrega ACL, Paula KC, Carvalho AC. Interaction between resistance training and flexibility training in healthy young adults. J Strength Cond Res 2005;19:842-6.

13. Banz WJ, Maher MA, Thompson WG, Bassett DR, Moore W, Ashraf M, et al. Effect of resistance versus aerobic training on coronary artery disease risk factors. Exp Biol Med 2003;228:434-40.

14. Prabhakaran, B, Dowling, EA, Branch, JD, Swain, DP, Leutholtz, BC. Effects of 14 weeks of resistance training on lipid profiles and body fat percentage in premenopausal women. Br J Sports Med 1999:33:190-5.
15. Bemben DA, Bemben MG. Effects of resistance exercise and body mass index on lipoprotein-lipid patterns of postmenopausal women. J Strength Cond Res 2000;14:80-5.

16. Honkola A, Forsén T, Eriksson J. Resistance training improves the metabolic profile in individuals with type 2 diabetes. Acta Diabetol 1997;34:245-8

17. Maiorana A, O'Driscoll G, Goodman C, Taylor R, Green D. Combined aerobic and resistance exercise improves glycemic control and fitness in type 2 diabetes. Diabetes Res Clin Pract 2002; 56:115-123.

18. Staron RS, Murray TF, Guilders RM, Hagerman FC, Hikida RS, Ragg K.E. Influence of resistance training on serum lipid and lipoprotein concentrations in young men and women. J Strength Cond Res 2000;14:37-44.

19. Smutok MA, Reece C, Kokkinos PF, Farmer C,Dawson P, Shulman R, et al. Aerobic versus strength training for risk factor intervention in middle-aged men at high risk for coronary heart disease. Metabolism 1993;42:177-84.

20. Fahlman MM, Boardley D, Lambert CP, Flynn MG. Effects of endurance training and resistance training on plasma lipoprotein profiles in elderly women. J Gerontol A Biol Sci Med Sci 2002;57:54-60.

21. Elliot KJ, Sale C, Cable NT. Effects of resistance training and detraining on muscle strength and blood llipid profiles in postmenopausal women. Br J Sports Med 2002;36:340-44.

22. Tsuzuku S, Kajioka T, Endo H, Abbott RD, Curb JD, Yano K. Favorable effects of non-instrumenta resistance training on fat distribution and metabolic profiles in healthy elderly people. Eur J App Physiol 2007;99:549-55.

23. Yarasheski KE, Tebas P, Stanerson B, Claxton S, Marin D, Bae K, et al. Resistance exercise training reduces hypertriglyceridemia in HIV-infected men treated with antiviral therapy. J Appl Physiol 2001;90:133-8.

24. Kelley GA, Kelley KS. Impact of progressive resistance training on lipids and lipoproteins in adults: a meta-analysis of randomized controlled trials. Prev Med 2009;48:9-19.

25. Williams MA, Haskell WL, Ades PA, Amsterdam EA, Bittner V, Franklin BA, et al. Resistance exercise in individuals with and without cardiovascular disease: 2007 update: a scientific statement from the American Heart Association Council on Clinical Cardiology and Council on Nutrition, Physical Activity, and Metabolism. Circulation 2007:116:572-84

26. Ferguson MA, Alderson NL, Trost SG, Essig DA, Burke JR, Durstine JL. Effects of four different single exercise sessions on lipids, lipoproteins, and lipoprotein lipase. J Appl Physiol 1998;85:1169-74.

27. Hickson JF, Wolinsky I. Nutrição no exercício e no esporte. 2a. ed. São Paulo: Roca, 2002.

28. Hokanson JE, Austin MA. Plasma triglyceride level is a risk factor for cardiovascular disease independent of high-density lipoprotein cholesterol level: a meta-analysis of population-based prospective studies. J Cardiovasc Risk 1996:3:213-19. 